\title{
Immunoglobulin and complement deposition in glomeruli of 756 subjects who had committed suicide or met with a violent death
}

\author{
J Varis, I Rantala, A Pasternack, H Oksa, M Jäntti, E S Paunu, R Pirhonen
}

Institute of Clinical Sciences, University of Tampere, Finland J Varis

A Pasternack

H Oksa

M Jäntti

E S Paunu

R Pirhonen

Department of

Pathology, Tampere University Hospital I Rantala

Correspondence to: Juha Varis, Institute of Clinical Sciences, University Clinical Sciences, University of Tampere, Box 607, SF
33101 Tampere, Finland Accepted for publication 3 February 1993

\begin{abstract}
Aims-To study immune deposits in renal glomeruli.

Methods-Tissue was obtained from 756 necropsy cases from people who had committed suicide or met with a violent death. Glomerular immune deposits were examined by immunofluorescence microscopy and a light microscopy. The clinical histories of all the deceased were studied to ascertain reasons for the deposits.
\end{abstract}

Results-Immune deposits were found in glomeruli in 91 (12\%) cases. In $52(6.8 \%)$ cases mesangial IgA was observed as a solitary finding in $34(4 \cdot 5 \%)$, and was accompanied by other immunoglobulins in $18(2 \cdot 4 \%)$. Mes-angial IgM was present in $19(2 \cdot 5 \%)$ and IgG in 11 cases $(1 \cdot 5 \%)$. Two cases had capillary IgG $(0 \cdot 3 \%)$. Light microscopic examination showed mesangial enlargement in eight of the cases with mesangial IgA. These included one with IgA glomerulonephritis diagnosed before death. Two cases with normal glomerular morphology and mesangial IgA deposits had clinical laboratory evidence of renal disease. In two subjects with normal glomerular morphology, mesangial IgM and microscopic haematuria were present. In one case with capillary IgG membranous glomerulonephritis was detected.

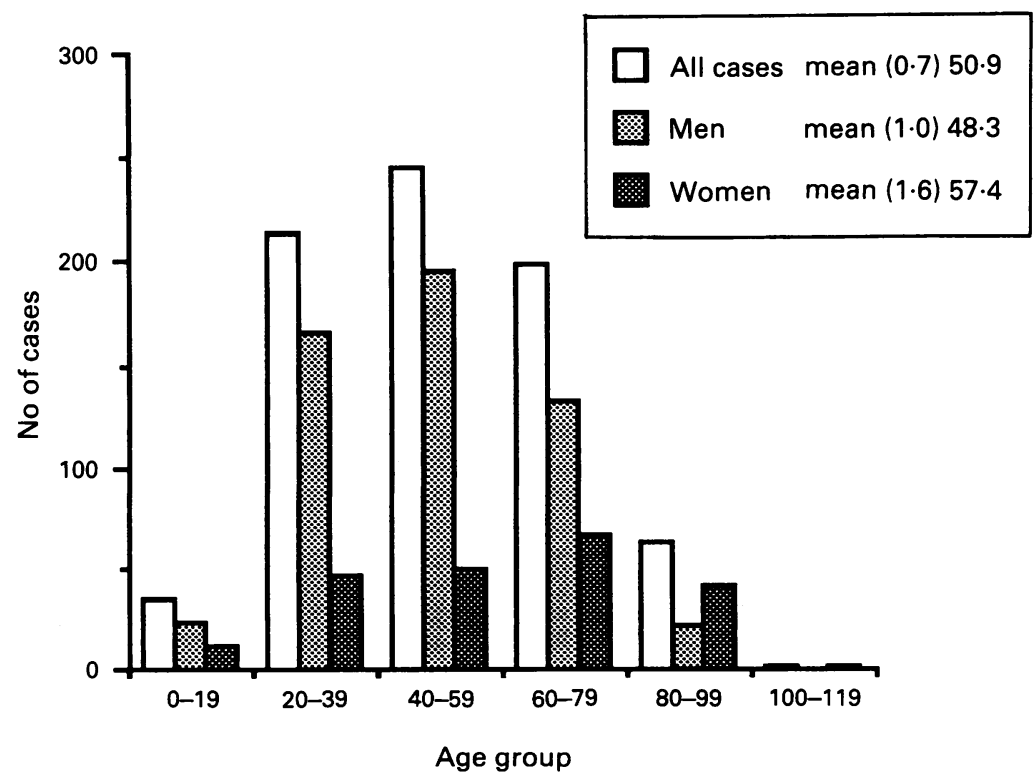

Figure 1 Age and sex distribution of study material.
Conclusions-Ten cases had mesangial IgA together with morphological or clinical laboratory findings suggestive of renal disease. If all these are regarded as IgA glomerulonephritis, then its prevalence can be estimated at $1 \cdot 3 \%$. For IgM glomerulonephritis, a prevalence of $0.3 \%$ was deduced.

\section{(F Clin Pathol 1993;46:607-610)}

Glomerular immune deposits can occur without clinical evidence of glomerulonephritis. ${ }^{12}$ The prevalence of these deposits is surprisingly high: $4 \%$ for mesangial IgA and $1 \%$ for IgM in unselected necropsy material from Singapore ${ }^{1}$; and $4.8 \%$ for IgA deposits in a hospital necropsy study from Germany. ${ }^{2}$ Biopsy specimens from cadaveric renal transplants have also shown glomerular deposits in $10.9 \%$ of cases studied on immunofluorescence. ${ }^{3}$ Not much is known about the reasons why immunoglobulins are deposited.

\section{Methods}

Over three years (1985-1987) 1813 forensic necropsies (540 men and 216 women) were performed at Tampere University Hospital. In 756 cases death had been caused by violent death or suicide. The age distribution by sex is shown in fig 1 .

Specimens of cortical kidney tissue for immunofluorescence and light microscopy studies were obtained at necropsy from all of the deceased. Eighty per cent of cases were necropsied within seven days of death, and $99 \%$ within 16 days of death. We have already shown that immunofluorescence from immunoglobulins and complement in necropsy tissue can be reliably interpreted up to 17 days after death. ${ }^{4}$

For immunofluorescence studies, the specimens were embedded in Ames OCT compound and frozen in liquid nitrogen. The frozen tissue was cut, fixed in absolute acetone, and stained with fluorescein isothiocyanate (FITC) conjugated antisera to human IgA, IgG, IgM, C1q, and C3b. Controls included incubation in FITC conjugated non-specific antiserum and incubation in an unconjugated blocking antiserum before the FITC-conjugate was used. All antisera were obtained from Behringwerke AG (Marburg-Lahn, Germany).

For light microscopy, the tissue was routinely embedded in paraffin wax. The speci- 
Table 1 Location and appearance of different immune reactants in relation to predominant imunofluorescence finding

\begin{tabular}{|c|c|c|c|c|c|c|}
\hline $\begin{array}{l}\text { Predominant } \\
\text { Immunofluorescence } \\
\text { finding }\end{array}$ & $\operatorname{Ig} A$ & $\operatorname{Ig} G$ & $I g M$ & $C 1 q$ & $C 3 b$ & $N=$ \\
\hline IgA & $\begin{array}{l}M G \\
M G \\
M G \\
M G \\
M G \\
M G \\
M G \\
M G\end{array}$ & $\begin{array}{l}M G \\
M G \\
M G\end{array}$ & $\begin{array}{l}M G \\
M G \\
M G\end{array}$ & $\begin{array}{l}M G \\
M G\end{array}$ & $\begin{array}{l}\text { M G } \\
M G\end{array}$ & $\begin{array}{r}34 \\
5 \\
4 \\
3 \\
1 \\
2 \\
1 \\
1\end{array}$ \\
\hline IgG & M G & $\begin{array}{l}M G \\
C G \\
M G \\
M G \\
M G \\
C G\end{array}$ & $\begin{array}{l}M G \\
C G\end{array}$ & $\begin{array}{l}M G \\
M G \\
M G\end{array}$ & & $\begin{array}{l}6 \\
1 \\
3 \\
1 \\
1 \\
1\end{array}$ \\
\hline IgM & & M G & $\begin{array}{l}M G \\
M G \\
M G\end{array}$ & M G & & $\begin{array}{r}17 \\
1 \\
1\end{array}$ \\
\hline $\mathrm{C} 1 \mathrm{q}$ & & & & M G & & 5 \\
\hline $\mathrm{C} 3 \mathrm{~b}$ & & & & & M G & 2 \\
\hline
\end{tabular}

C G = granular fluorescence along capillary walls, M G = mesangial and granular fluorescence.

Figure 2 Cases with immunofluorescence findings: their light microscopic findings and clinical data. mens with positive immunofluorescence findings were stained by haematoxylin and eosin, periodic acid Schiff, and Jones's periodic acid silver-methenamine methods.
1813 forensic necropsies
Clinical data were collected from necropsy documents, the files of Tampere University Hospital, and the Discharge Reporting System. This register, organised by the Finnish National Board of Health, covers all hospitals in the country. The local Pirkanmaa Kidney Register was also examined. By combining data from these sources, clinical information was available for 590 (78\%) cases. The correlations between immunofluorescence findings and the diseases encountered were analysed using the $\chi^{2}$ test.

\section{Results}

Of the 756 necropsies, immunofluorescence showed renal deposits of immunoglobulin in $91(12 \%)$ (table 1). There was no correlation between the prevalence of positive immunofluorescence findings and the age or sex of the deceased. The immune deposits were granular, diffusely distributed, and located in mesangial areas except in two cases with capillary IgG (fig 2).

Mesangial IgA was found in $52(6.9 \%)$ cases. Isolated IgA deposits were present in
Earlier confirmed IgA GN:

1 case

9 cases
Possible IgA GN:

756 traumatic deaths; 540 men and 216 women

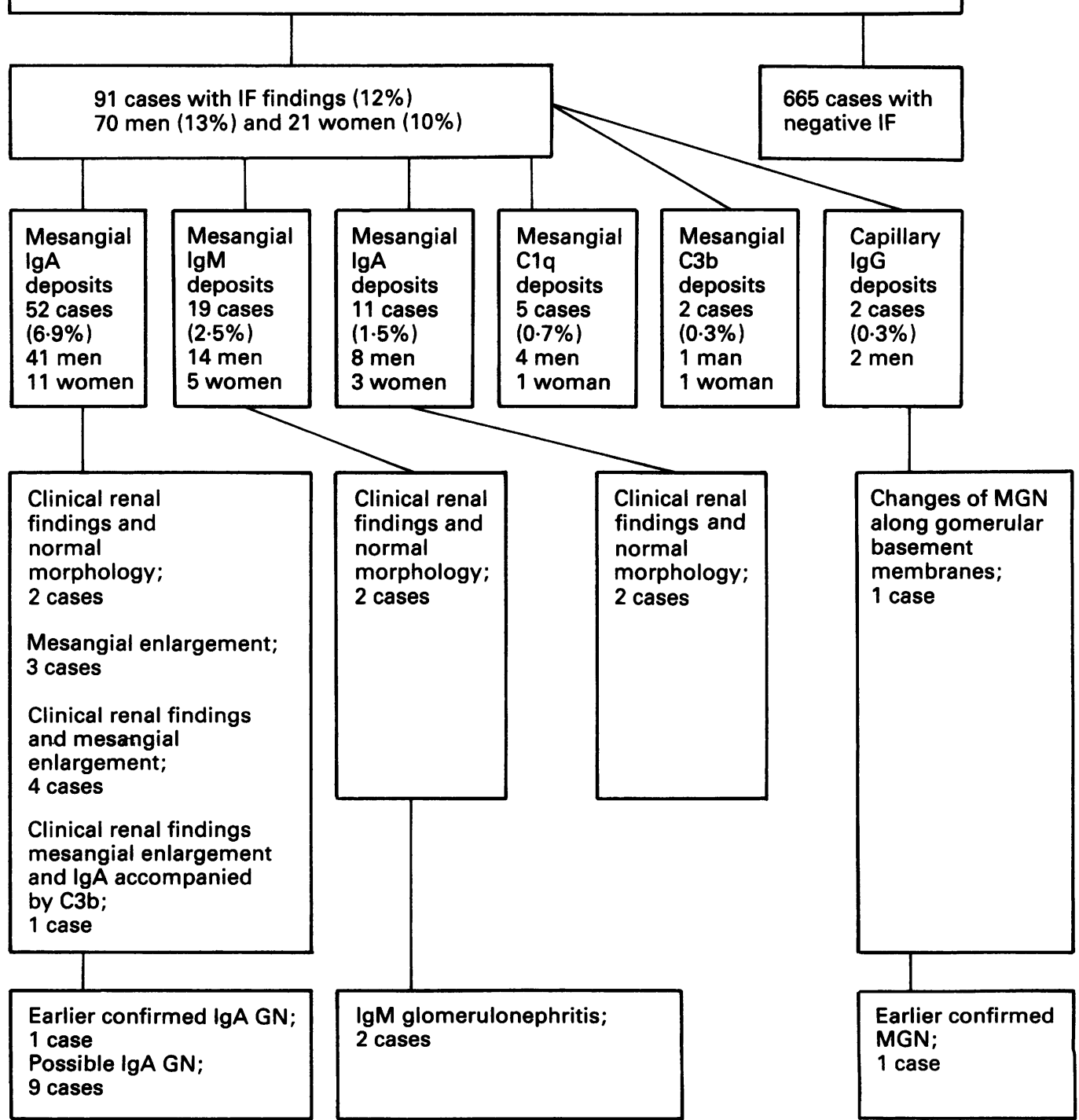


Table 2 Data on previous clinical finding in the kidney in 38 cases

\begin{tabular}{|c|c|c|c|c|}
\hline \multirow{2}{*}{$\begin{array}{l}\text { Renal } \\
\text { diseases }\end{array}$} & \multirow{2}{*}{$\begin{array}{l}\text { Cases in total } \\
\text { study population }\end{array}$} & \multicolumn{3}{|c|}{ Cases with immunofluorescence deposits } \\
\hline & & $\operatorname{Ig} A$ & $\operatorname{Ig} M$ & $\operatorname{Ig} G$ \\
\hline Renal cysts & 13 & - & - & - \\
\hline Renal trauma & 6 & 1 & - & - \\
\hline Chronic pyelonephritis & 6 & - & - & 1 \\
\hline Hydronephrosis & 2 & 1 & - & - \\
\hline Kidney anomaly & 2 & 1 & - & - \\
\hline Interstitial nephropathy & 3 & - & - & - \\
\hline Glomerulonephritist & 2 & 1 & - & 1 \\
\hline Unknown nephropathy & 2 & - & - & - \\
\hline Renal scars in urography & 1 & - & - & - \\
\hline Renal calculi & 1 & - & - & - \\
\hline
\end{tabular}

^Two cases with rhabdomyolysis and transient failure during life and one with unknown interstital nephropathy.

fOne case with IgA glomerulonephritis and one with mesangial and granular fluorescence, both fOne case with IgA glomerulonephritis and one with mesangial and granular fluorescence, both
with pre-mortem diagnosis. In three cases two renal diseases were detected in the same person; with pre-mortem diagnosis. In three cases two renal diseases were detected in the same person;
one with glomerulonephritis and duplex ureter, one with chronic pyelonephritis and slight hydronephrosis, and one with hydronephrosis and renal cysts. The disease mentioned first was regarded as the primary diagnosis.

$34(4 \cdot 5 \%)$ specimens. IgA was accompanied by complement in four cases, and by other immunoglobulins in 14 (table 1).

Of the cases with mesangial IgA (fig 2), light microscopic examination showed mesangial enlargement in eight. In two other cases previous clinical data had indicated renal disease but this was not detected at light microscopy. Of these 10 cases one had IgA glomerulonephritis diagnosed before death. If the remaining nine diagnoses are also taken to be IgA glomerulonephritis, on the basis of the diffuse and global occurrence of IgA, together with morphological or clinical laboratory signs of renal disease (fig 2), a prevalence of $1.3 \%$ for IgA glomerulonephritis can be deduced.

Mesangial IgM was found in $19(2.5 \%)$ and IgG in $11(1.5 \%)$ cases. Isolated $\mathrm{Clq}$ was present in five $(0.7 \%)$ and $\mathrm{C} 3 \mathrm{~b}$ in two $(0.3 \%)$ cases (table 1$)$. In all of these specimens the glomerular morphology was normal.

Two cases with IgM deposits had microscopic haematuria (fig 2). According to current opinion, these represent cases of IgM glomerulonephritis. This gives a prevalence of $0.3 \%$ for IgM glomerulonephritis.

Granular IgG deposits along the capillary basement membranes were seen in two cases $(0.3 \%)$. One of these had heavy proteinuria, and membranous glomerulonephritis was seen at light microscopy.

Previous data on clinical renal findings were found in 38 cases (table 2).

Among the 590 cases with available clinical data the percentage of positive immunofluorescence findings was $11.7 \%$; in the remaining 166 cases it was $13 \cdot 2 \%$. There were no differences in the prevalence of positive immunofluorescence findings among subjects known to have had a variety of non-glomerular diseases.

\section{Discussion}

We investigated the occurrence of glomerular deposits of immunoglobulins and complement by immunofluorescence in material selected from the population on the basis of sudden death. There were more men, with a predominance of middle-aged men and old women (fig 1). In our study there was a delay between death and necropsy. We have already shown, however, that immunofluorescence can reliably show the presence of glomerular immunoglobulins and complement up to 17 days after death. ${ }^{4}$ At light microscopy, autolysis was evident but it did not affect the interpretation of glomerular morphology. In fact, the two cases with biopsy confirmed glomerulonephritis (IgA glomerulonephritis, membranous glomerulonephritis) were also diagnosable after death on the basis of typical imınunofluorescence and light microscopic findings.

Sinniah has described immunofluorescence results in tissue from subjects who had met with a violent death, ${ }^{1}$ and so has Waldherr et al in patients at necropsy. ${ }^{2}$ Sinniah found mesangial IgA to be present in 4\%: Waldherr and coworkers detected IgA in $4.8 \%$ of the necropsied patients. Curschellas et al found glomerular IgA in $6.9 \%$ of biopsy specimens from cadaveric grafts studied by immunofluorescence. ${ }^{3}$ These results agree with those of our study. The occurrence of glomerular IgA seems to be high in various populations. Deposition is probably not inevitably associated with any clinically apparent condition. If the cases with glomerular $\operatorname{IgA}$, together with those that had morphological or clinical laboratory findings of renal disease, are regarded as IgA glomerulonephritis, a prevalence of $1.3 \%$ for IgA glomerulonephritis can be estimated.

In IgA glomerulonephritis IgA is almost invariably accompanied by complement deposition, ${ }^{5}$ which has been considered to be of central importance in the pathogenesis of both human and experimentally induced IgA glomerulonephritis. ${ }^{67}$ On the other hand, the quality of antigen is thought to have a critical role in the development of glomerulonephritis associated with IgA immune complexes. ${ }^{8}$ The IgA deposits found in this study were mostly solitary. Isolated IgA deposits have also been shown to occur in coeliac disease, ${ }^{9}$ liver diseases, ${ }^{10}$ and neoplastic diseases. ${ }^{11}$ In the study by Curschellas et al complement was detected along with IgA in only one out of seven cases with glomerular IgA. ${ }^{3}$ In our electron microscopic studies electron-dense deposits were found in mesangial areas and they were morphologically similar to those seen in IgA glomerulonephritis.

These results suggest that IgA can be deposited mesangially without leading to overt glomerular damage. The deposits probably do not do any damage until some as yet undefined circumstance induces clinically apparent glomerulonephritis. Complement seems to be one of the linking factors between subclinical IgA deposition and IgA glomerulonephritis.

Sinniah found IgM deposits in $1 \%$ of his material. ${ }^{1}$ The corresponding figure in our study was $2.5 \%$. Although mesangial IgM deposition is a common non-specific phenomenon found in immunofluorescence studies, two cases with microscopic haematuria diagnosed before death were regarded as IgM 
glomerulonephritis. ${ }^{12}$ In our study IgA glomerulonephritis occurred five times as often as IgM glomerulonephritis. This finding agrees with that of Mustonen et $a l,{ }^{13}$ who found that the ratio of $\operatorname{IgA}$ glomerulonephritis to IgM glomerulonephritis was 3.4.

As to the other immunofluorescence findings, they seemed to be randomly distributed among various clinical conditions. One case with capillary IgG deposits proved to be membranous glomerulonephritis. On the other hand, the presence of other immune reactants remained unexplained on the basis of the available background data.

1 Sinniah R. Occurrence of mesangial IgA and IgM deposits in a control necropsy population. F Clin Pathol 1983, 36:276-9.

2 Waldherr $\dot{R}$, Rambausek $M$, Duncker WD, Ritz E. Frequency of mesangial IoA deposits in a non-selected autopsy series. Nephrol Dial Transplant 1989;4:943-6.
3 Curschellas E, Landmann J, Durig M, et al. Morphologic findings in "zero-hour" biopsies of renal transplants. Clin Nephrol 1991;36:215-22.

4 Varis J, Rantala I, Pasternack A. Immunofluorescence of immunoglobulins and complement in kidneys taken at necropsy. F Clin Pathol 1989;42:1211-4.

5 van der Peet J, Arisz L, Brentjens JRH, Marrink J, Hoedemaeker PJ. The clinical course of IgA nephropaHoedemaeker PJ. The clinical course of

6 Emancipator SN, Lamm ME. IgA nephropathy: pathogenesis of the most common form of glomerulonephri-
gancipator SN, Lamm ME. IgA nephropathy: pathotis. Lab Invest 1989;60:168.

7 Emancipator SN, Ovary Z, Lamm ME. The role of mesangial complement in the hematuria of experimental IgA nephropathy. Lab Invest 1987;57:269.

8 Montinaro V, Esparza AR, Cavallo T, Rifai A. Antigen as mediator of glomerular injury in experimental IgA nephropathy. Lab Invest 1991;64:508-19.

9 Pasternack A, Collin P, Hustonen J, et al. Glomerular IgA deposits in patients with coeliac disease. Clin Nephrol 1990;34:56-60.

10 Berger J, Yaneva H, Nabarra B. Glomerular changes in patients with cirrhosis of the liver. Adv Nephrol 1978;7: patients

11 Mustonen J, Pasternack A, Helin H. IgA mesangial nephropathy in neoplastic diseases. Contrib Nephrol 1984;40:283-91.

12 Helin H, Mustonen J, Pasternack A, Antonen J. IgMassociated glomerulonephritis. Nephron 1982;31:11-6.

13 Mustonen J, Rantala I, Helin H, Pasternack A. IgA-IgM nephropathy; a subgroup of primary mesangial glomerulonephritis. Am f Clin Pathol 1991;95:107-10. 